\title{
Angular dependence of giant Zeeman effect for semimagnetic cavity polaritons
}

\author{
R. Mirek, ${ }^{1,{ }^{*}}$ M. Król, ${ }^{1}$ K. Lekenta, ${ }^{1}$ J.-G. Rousset, ${ }^{1}$ M. Nawrocki, ${ }^{1}$ M. Kulczykowski, ${ }^{2}$ M. Matuszewski, ${ }^{2}$ \\ J. Szczytko, ${ }^{1}$ W. Pacuski, ${ }^{1}$ and B. Piętka ${ }^{1, \dagger}$ \\ ${ }^{1}$ Institute of Experimental Physics, Faculty of Physics, University of Warsaw, ulica Pasteura 5, PL-02-093 Warsaw, Poland \\ ${ }^{2}$ Institute of Physics, Polish Academy of Sciences, Aleja Lotników 32/46, PL-02-668 Warsaw, Poland \\ (Received 29 August 2016; revised manuscript received 20 December 2016; published 21 February 2017)
}

\begin{abstract}
The observation of spin-related phenomena of microcavity polaritons has been limited due to the weak Zeeman effect of nonmagnetic semiconductors. We demonstrate that the incorporation of magnetic ions into quantum wells placed in a nonmagnetic microcavity results in enhanced effects of magnetic field on exciton-polaritons. We show that in such a structure the Zeeman splitting of exciton-polaritons strongly depends on the photon-exciton detuning and polariton wave vector. Our experimental data are explained by a model where the impact of magnetic field on the lower polariton state is directly inherited from the excitonic component, and the coupling strength to the cavity photon is modified by an external magnetic field.
\end{abstract}

DOI: 10.1103/PhysRevB.95.085429

\section{INTRODUCTION}

Cavity exciton-polaritons are quasiparticles resulting from the strong coupling between cavity optical modes and exciton resonances. The most frequently studied system consists of an exciton confined in a quantum well embedded in a semiconductor microcavity. During the last decade, intensive research showed that such a system is a well-suited playground for investigating fundamental objects like Bose-Einstein condensates [1,2] or polariton superfluids [3,4], making the physics of nonequilibrium quantum fluids accessible at higher temperatures $[5,6]$. The unique features of polariton condensates set the basis for new polaritonic devices [7,8].

In recent years an interest in the magneto-optical properties of cavity polaritons has risen [9-18]. In the extensively studied GaAs-based microcavities the polariton Zeeman splitting is of the order of the emission linewidth in magnetic fields easily accessible in laboratories [16]. However, semimagnetic semiconductors offer the opportunity to enhance magnetooptical effects via the exchange interaction between the $d$ shell electrons of a magnetic ion and the $s$-shell electrons and $p$-shell holes of the conduction and valence bands of the host material [19]. This $s, p$ - $d$ exchange interaction leads to enhanced magneto-optical effects like giant Faraday rotation [20] or giant Zeeman splitting [21]. At the same time, the incorporation of magnetic ions can lead also to photoluminescence (PL) quenching and an important lowering of optical quality, which is particularly striking in the case of $\mathrm{Ga}_{1-x} \mathrm{Mn}_{x} \mathrm{As}$ structures [22,23]. Another type of material is a semiconductor system relatively resistant to manganeseinduced optical degradation: $\mathrm{Cd}_{1-x} \mathrm{Mn}_{x} \mathrm{Te}$, which was already used as a high-refractive-index layer in microcavities with $\mathrm{Cd}_{1-x} \mathrm{Mn}_{x}$ Te quantum wells [24,25]. Indeed such a system exhibits strong magneto-optical properties [26-28] related to the shift of both exciton and photon states [24,29]; therefore, it can be used for tuning the exciton-photon energy difference and for tuning the Rabi oscillation frequency [30-32].

\footnotetext{
*rafal.mirek@student.uw.edu.pl

†barbara.pietka@fuw.edu.pl
}

Our approach to semimagnetic cavity polaritons is based on redesigned structures where magnetic ions are inserted only in the quantum wells, while the cavity and the distributed Bragg reflectors (DBRs) are made of nonmagnetic materials $[33,34]$. We show that in such a case the giant Zeeman effect of polaritons results only from the strong coupling of cavity photons with semimagnetic excitons confined in CdTe quantum wells containing manganese ions.

The giant Zeeman splitting of polaritons is studied in angleresolved photoluminescence and reflectivity experiments. A theoretical model taking into account the dependence of the splitting on the in plane wave vector and the photon-exciton detuning is described. These results are the first step towards the study of spinor polariton condensates [12,35,36] with enhanced magneto-optical properties.

\section{SAMPLE AND EXPERIMENTAL SETUP}

The sample studied in this work was grown using molecular beam epitaxy. On a (100)-oriented GaAs substrate a $2-\mu$ m-thick CdTe buffer is followed by a 780-nm-thick $\mathrm{Cd}_{0.86} \mathrm{Zn}_{0.14} \mathrm{Te}$ buffer. Starting from this buffer layer, the whole structure is lattice matched to MgTe [37,38]. Photons are confined by DBRs made of $\mathrm{Cd}_{0.77} \mathrm{Zn}_{0.13} \mathrm{Mg}_{0.10} \mathrm{Te}$ for the high-refractive-index layers and $\mathrm{Cd}_{0.43} \mathrm{Zn}_{0.07} \mathrm{Mg}_{0.50} \mathrm{Te}$ for the low-refractive-index layers. Respective refractive indices [34,37] at the center wavelength $\lambda_{0}=735 \mathrm{~nm}$ are $n_{\text {high }}=$ 2.97 and $n_{\text {low }}=2.61$. Four 20-nm-wide $\mathrm{Cd}_{0.83} \mathrm{Zn}_{0.16} \mathrm{Mn}_{0.01} \mathrm{Te}$ quantum wells (QWs) are placed at the antinodes of the electric field of the $3 \lambda$ cavity. Since our structure is designed to enhance magneto-optical effects in cavity polaritons only via the excitonic component, manganese is incorporated only in quantum wells, not in the surrounding material or in the DBRs. The detailed scheme of the sample structure is shown in Fig. 1.

The sample is placed in a magnetic field up to $5 \mathrm{~T}$ in a Faraday configuration on the cold finger of an optical cryostat at the temperature of $10 \mathrm{~K}$. The sample is excited nonresonantly by a continuous-wave Ti:sapphire laser for which the energy is tuned to the first reflectivity minimum of the cavity stop-band on the high-energy side: $E_{\text {exc }}=1.76 \mathrm{eV}$ $\left(\lambda_{\text {exc }}=705 \mathrm{~nm}\right)$. The angle-resolved photoluminescence and reflectivity spectra not resolved in polarization are collected, 


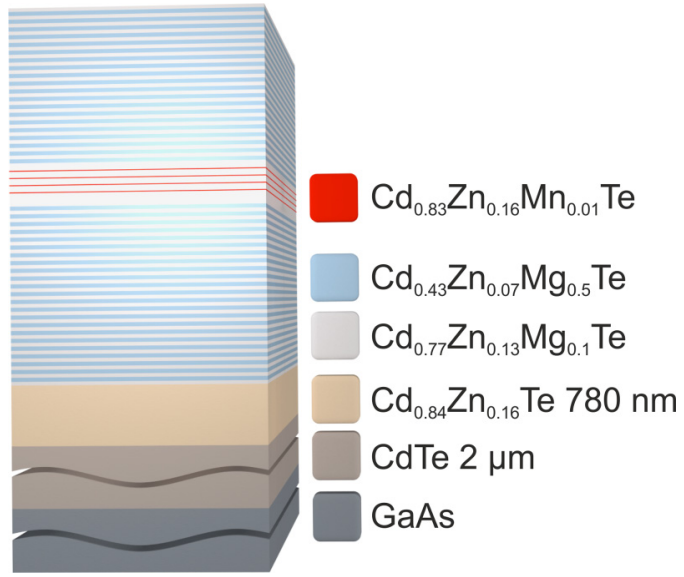

FIG. 1. The structure of the microcavity sample: four 20 -nm-wide QWs (red) containing $\mathrm{Mn}^{2+}$ ions sandwiched between 23 layers (bottom) and 20 layers (top) nonmagnetic distributed Bragg reflectors (alternating blue and white layers). Semimagnetic cavity polaritons result from the strong coupling of cavity photons with excitons confined in the semimagnetic QWs.

giving direct information about the polariton dispersion (energy-wave vector dependence), with the wave-vector scale being proportional to the angle at which light is emitted from the structure [1].

\section{EXPERIMENTAL RESULTS: IMAGING POLARITON DISPERSION BY PHOTOLUMINESCENCE AND REFLECTIVITY}

Figure 2 illustrates the evolution of the exciton-polariton dispersion in a magnetic field for two representative cases of negative (top) and positive (bottom) photon-exciton detunings.
The detuning is defined as the difference in energy between the bare cavity mode and the exciton energy at zero emission angle, $\delta=E_{\mathrm{ph}}-E_{\mathrm{exc}}$, where $E_{\mathrm{ph}}$ and $E_{\mathrm{exc}}$ are photon and exciton energies, respectively. At the magnetic field the detuning is defined separately for the two Zeeman-split polariton components. The maps show PL spectra measured as a function of the emission angle, for zero magnetic field (left-hand panels) and for magnetic fields increasing up to $5 \mathrm{~T}$ (right-hand panels). The Zeeman splitting of the lower polariton state increases with magnetic field, which is very well visible for positive detuning, where the excitonic contribution to the polariton wave function is dominant. Interestingly, also for negative detuning, i.e., photonlike lower polariton states, a significant splitting can be observed at high magnetic field (top-right panel). However, in this case, the splitting is visible for high emission angles. Since in a first approximation the magnetic field affects only excitons, it does not change the photon states [16,39]; we explain the magnetic-field evolution of a photonlike polariton as a consequence of the exciton state contribution evolution with a $k$-vector, magnetic field, and detuning.

A more detailed analysis of the impact of photon-exciton detuning on the polariton dispersion is presented in Fig. 3, where sets of photoluminescence and reflectivity maps in a magnetic field of $5 \mathrm{~T}$, measured for various values of photon-exciton detuning from large negative (left side) to small positive (right side) detuning, are presented. Both photoluminescence and reflectivity measurements lead to a consistent image of the polariton dispersion. The first conclusion to be imposed is that the splitting is more visible for the lower polariton with a higher excitonic content. The splitting of photonlike polaritons (negative detuning) is visible only for large values of the wave vector and, at zero wave vector, the Zeeman splitting is smaller than the linewidth. In the positive detuning case, the energy difference between the split components of the lower polariton is more pronounced.

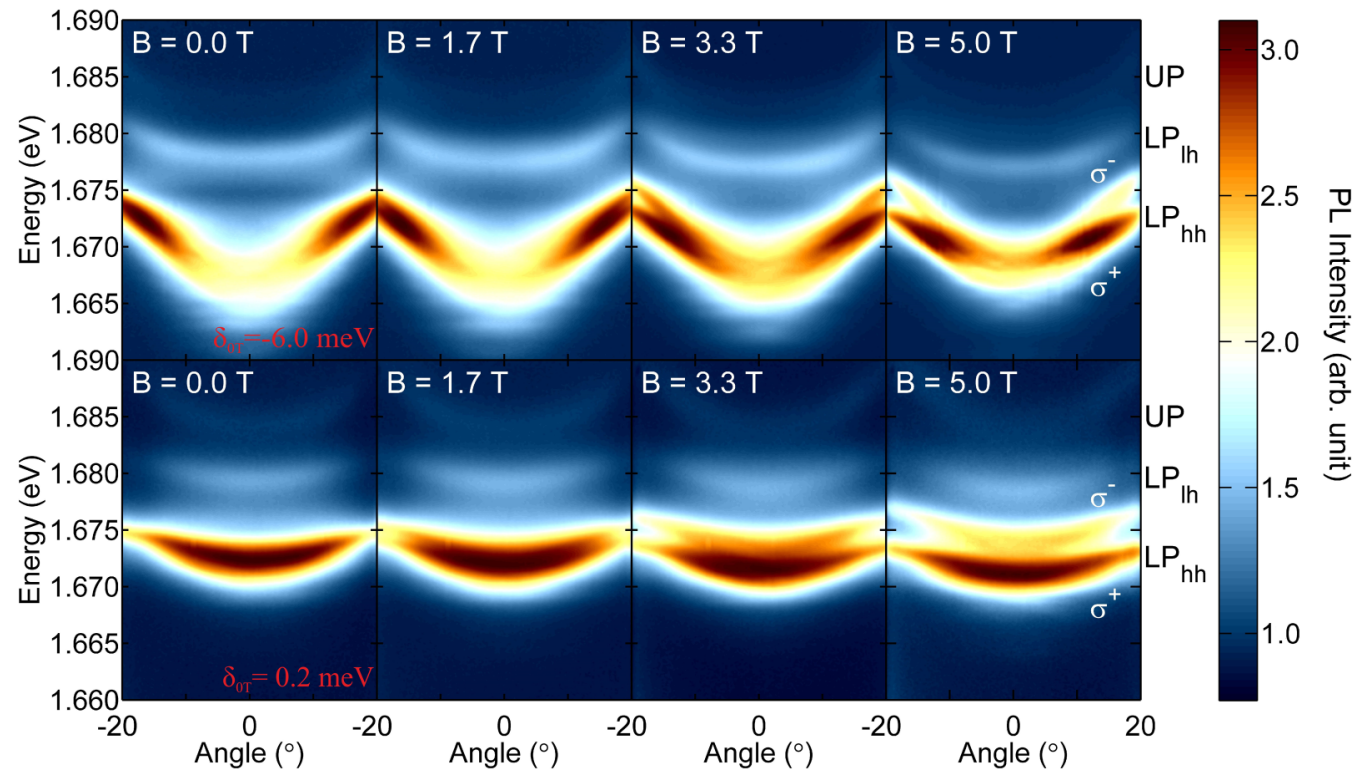

FIG. 2. Angle-resolved photoluminescence maps of semimagnetic exciton-polaritons for negative $\left(\delta_{0 \mathrm{~T}}=-6.0 \mathrm{meV}\right.$, top $)$ and slightly positive $\left(\delta_{0 \mathrm{~T}}=0.2 \mathrm{meV}\right.$, bottom) photon-exciton detuning (determined at zero emission angle) at magnetic fields of $0,1.7,3.3$, and 5.0 T. The giant Zeeman splitting is well resolved for positive detuning, but it is also observed for higher angles at negative detuning. 


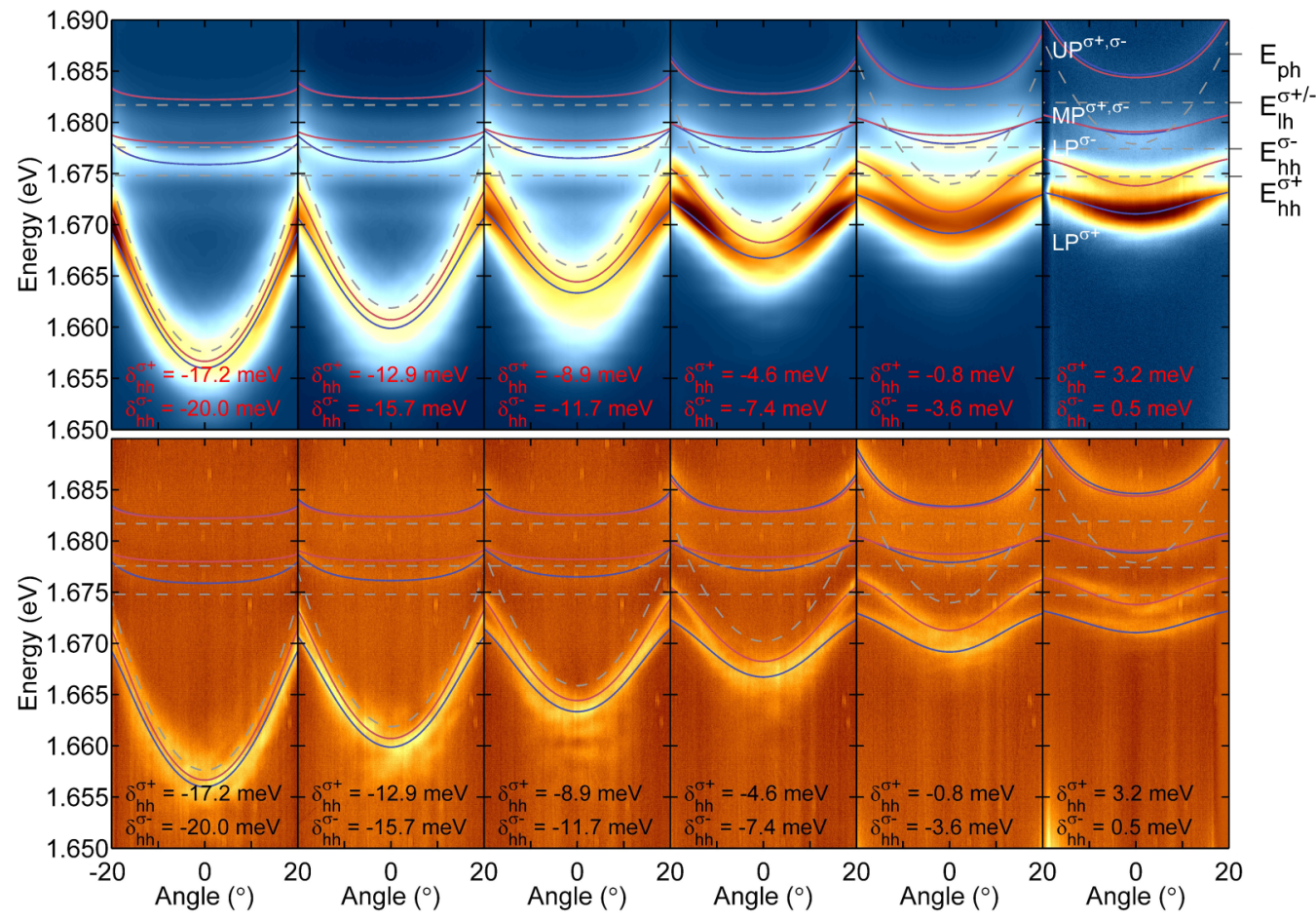

FIG. 3. Set of angle-resolved photoluminescence (top) and reflectivity (bottom) maps for different photon-exciton detuning (the detuning values are marked in the images for $\sigma^{+}$and $\sigma^{-}$polarization) in a magnetic field of $5 \mathrm{~T}$. Grey dashed curves indicate the calculated energies of the uncoupled exciton and photon. The energies of polariton branches are marked by red $\left(\sigma^{-}\right)$and blue $\left(\sigma^{+}\right)$curves.

Furthermore, it does not depend so strongly on the wave vector as in the case of negative detuning.

\section{MODELING POLARITON DISPERSION}

We derived a simple theoretical model to explain the behavior of exciton-polaritons in a magnetic field. Heavy hole excitons have a fourfold degeneracy of spin projection on the axis of the cavity: $\pm 2, \pm 1$. The excitons of a spin projection \pm 2 remain dark, not active with respect to light coupling or photon emission, and are neglected in the following consideration. The optically active excitons, with +1 and -1 spin degeneracy, couple to $\sigma^{+}$and $\sigma^{-}$circularly polarized light, respectively, forming polaritons with two spin projections. Therefore, in our model we consider two independent Hamiltonians for both circularly polarized subsystems, $\hat{H}^{ \pm}$. Moreover, in our QWs the heavy and light hole separation energy is small, and we observe a strong contribution to the polariton states from excitons formed with light holes. In CdMnTe-based QWs the Zeeman splitting of the light hole excitons is one order of magnitude smaller than the splitting for heavy hole excitons [21]. Nevertheless, we take into account the splitting of light holes in our model. Our coupling Hamiltonians take the following three-level model form, each one corresponding to the coupling of heavy and light hole excitons to $\sigma^{+}, \sigma^{-}$ circular polarization of light:

$$
\hat{H}^{ \pm}=\left(\begin{array}{ccc}
E_{\mathrm{ph}} & \frac{\hbar \Omega_{\mathrm{lh}}^{\sigma \pm}}{2} & \frac{\hbar \Omega_{\mathrm{hh}}^{\sigma \pm}}{2} \\
\frac{\hbar \Omega_{\mathrm{lh}}^{\sigma \pm}}{2} & E_{\mathrm{lh}}^{\sigma \pm} & 0 \\
\frac{\hbar \Omega_{\mathrm{hh}}^{\sigma \pm}}{2} & 0 & E_{\mathrm{hh}}^{\sigma \pm}
\end{array}\right),
$$

where $E_{\mathrm{ph}}$ is the cavity photon energy and the energies of light hole and heavy hole excitons are $E_{\mathrm{lh}}$ and $E_{\mathrm{hh}}$, respectively. The coupling energy between the corresponding excitons with cavity photons is marked as $\Omega_{\mathrm{lh}}$ and $\Omega_{\mathrm{hh}}$. The symbols $\sigma^{+}$and $\sigma^{-}$correspond to two Zeeman-split components of excitons. The energy of both circularly polarized cavity photons, $E_{\mathrm{ph}}$, is the same and it is expected to be independent of magnetic field; however, to verify this assumption we leave this parameter free in the fitting procedure of the model to the experimental data.

In the top panel of Fig. 3 we directly compare the experimental polariton dispersion curves with the theoretical model. Grey dashed curves illustrate the calculated energies of the bare photon and excitons. Red and blue curves give energies of the polaritons with $\sigma^{-}$and $\sigma^{+}$polarization. The fitting of the model to the photoluminescence experimental data allows one to obtain the bare exciton and photon energies as well as the Rabi energy in a magnetic field. Figure 4 illustrates the fitted parameters for the data from the positive detuning (last PL map of Fig. 3). Figure 4(a) illustrates the energy change of the bare photon and excitons in the magnetic field. We observe that the photon energy does not change significantly in the magnetic field, which confirms our previous assumption. In contrast, the heavy hole exciton splits significantly in the magnetic field.

In the mean-field theory, the Zeeman splitting of a heavy hole exciton in semimagnetic semiconductors resulting from $s, p-d$ exchange interaction scales with the magnetization of magnetic ions [19]. Therefore, for low magnetic ion content, it is proportional to the Brillouin function $B_{5 / 2}$ describing magnetization of noninteracting $\mathrm{Mn}$ ions of spin $5 / 2$ at the temperature of the experiment $(10 \mathrm{~K})$ [21]. The observed 

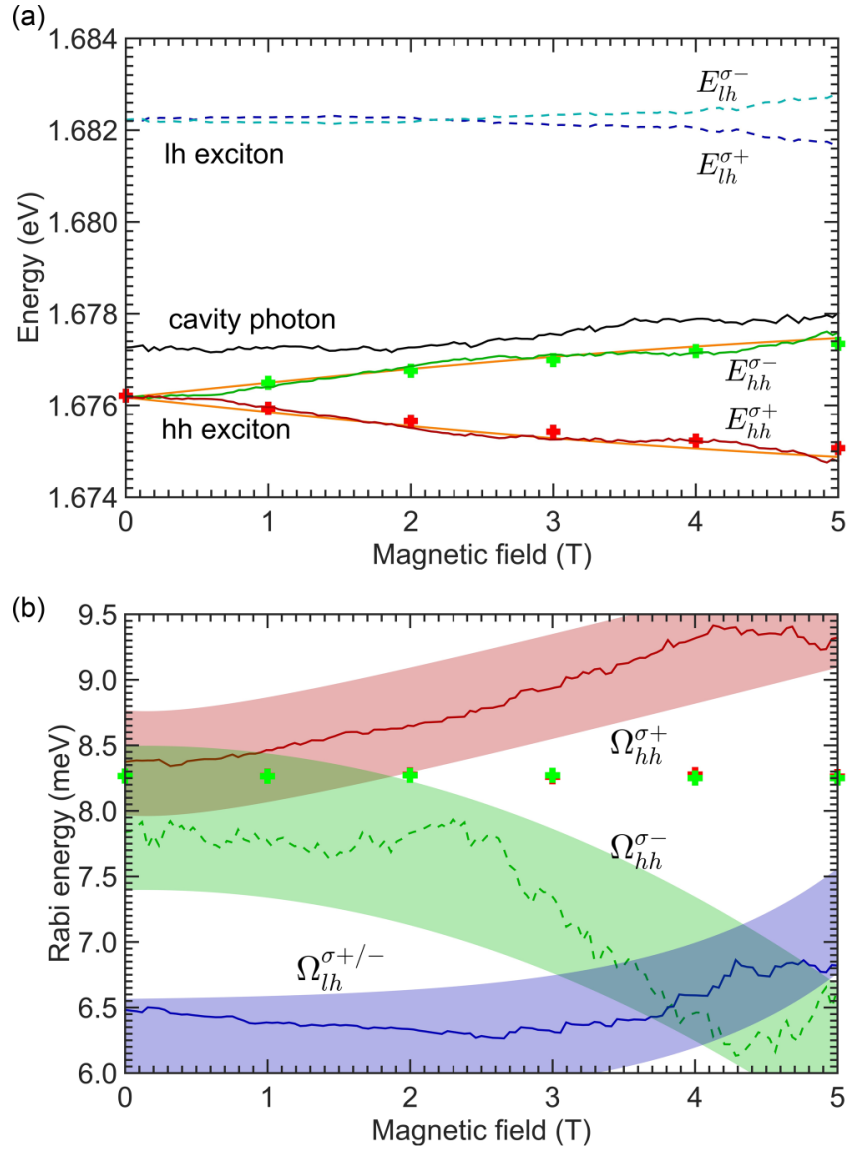

FIG. 4. (a) Energy change of heavy hole, $E_{\mathrm{hh}}$, light hole excitons, $E_{\mathrm{lh}}$, and a cavity photon induced by magnetic field. (b) Change of the Rabi energy in the magnetic field. The experimental results are obtained from the fitting of a solution of the Schrödinger equation with the Hamiltonian (1) to the data illustrated in the last panel of Fig. 3. The shading along the experimental curves represents the error margins of our fitting procedure. The dots illustrate the results of theoretical calculations based on a variational method applied to our structure.

almost linear dependence is due to the fact that measurements were performed at a temperature of about $10 \mathrm{~K}$, which agrees with the shape of the Brillouin function at this temperature, marked with an orange solid line in Fig. 4(a).

For better understanding of the magnetic field evolution of electrons and holes in our QW we calculated exciton energies by solving the full two-body Schrödinger problem using a variational method $[40,41]$. We assumed the exponential form of the relative electron-hole wave function and hyperbolic secant wave function for both electrons and holes in the direction perpendicular to the QW plane. We take into account the change of the exciton energy by calculating the band offset of electrons and holes as

$$
\begin{aligned}
& \Delta V_{e}^{\sigma_{ \pm}}= \pm N_{0} \alpha x\left\langle S_{z}\right\rangle, \\
& \Delta V_{\mathrm{hh}}^{\sigma_{ \pm}}=\mp N_{0} \beta x\left\langle S_{z}\right\rangle,
\end{aligned}
$$

where $\alpha$ and $\beta$ are the exchange interaction constants between magnetic ions and the electrons and holes, respectively, and $1 / N_{0}$ is the volume of the elementary crystal cell. We take into account bright excitons for which the spins of electrons and holes are aligned in opposite directions. The exchange integrals are taken according to [21] as $N_{0} \alpha=220 \mathrm{meV}$ and $N_{0} \beta=880 \mathrm{meV}$. As the ion concentration is very low, the mean spin is taken in the dilute limit as

$$
\left\langle S_{z}\right\rangle=\frac{5}{2} B_{5 / 2}\left(5 g \mu_{B} H / 2 k_{B} T\right)
$$

Here $\mu_{B}$ is the Bohr magneton, $g \approx 2$ is the $g$ factor of the ions, and $H$ is the strength of the external magnetic field in the Faraday configuration. The exciton $g$ factor of a nonmagnetic 20-nm-wide QW [42] ( $g=0.27)$ is small enough to be neglected. The theoretical energies shown in Fig. 4(a) with dots agree with experimental data when the sample background temperature $T=10 \mathrm{~K}$ is used to estimate the mean spin polarization. Moreover, taking into account the increased overlap of the electron and hole wave functions in the magnetic field and the energy shift of the electron and hole levels in the magnetic field (and, therefore, the different penetration of the wave function in the QW barrier), we calculated the effect of the magnetic field on the exciton oscillator strength and on the exciton binding energy. We found that such a magnetic-field-induced dependence is negligible in our structure and consequently predicted Rabi energy [shown by dots in Fig. 4(b)] is almost constant in the magnetic field. It is in contrast to the previous study of (Cd,Mn)Te/(Cd,Mg)Te QWs [41], but the difference is fully explained by relatively low $\mathrm{Mn}$ concentration in the QW and the strength of the confining potential, which both cause the perpendicular electron and hole wave functions in our structure to remain practically unaffected by the magnetic field. Prediction of almost constant Rabi energy is also different than in the case of GaAs-based microcavities, where the Rabi energy and exciton oscillator strength increase in the magnetic field [16], but in II-VI semiconductors exciton size is much smaller and consequently a direct effect of the magnetic field is much weaker.

Experimentally, by fitting the model described by Hamiltonian (1) to the observed emission lines, we observe that the Rabi energy of the heavy hole $\sigma^{+}$polariton slightly increases in the magnetic field and, interestingly, the Rabi energy of the heavy hole $\sigma^{-}$polariton is observed to decrease in the magnetic field. The comparison of the results of the fitting with different parameters for constant and varied Rabi energy in a magnetic field is illustrated in the Supplemental Material [43], where we demonstrate that the fitting to the experimental data is more accurate when the Rabi energy is a free parameter. The experimentally observed decrease of Rabi energy in $\Omega_{\mathrm{hh}}^{\sigma^{-}}$ is attributed to the effect of field-dependent inhomogeneous broadening of the exciton linewidth, due to fluctuations of Mn distribution in the QWs. Such broadening is known for semimagnetic semiconductors $[44,45]$. In zero magnetic field, the linewidth of the exciton emission line is related to the nonuniform distribution of $\mathrm{Mn}$ in the QW because the energy gap of CdMnTe increases with the manganese concentration. In a magnetic field the Zeeman splitting which increases with the $\mathrm{Mn}$ concentration is greater for excitons with higher energy at $B=0$ than for excitons with a smaller one, resulting in a narrowing of the exciton emission linewidth in $\sigma^{+}$polarization and broadening in $\sigma^{-}$polarization [43]. 


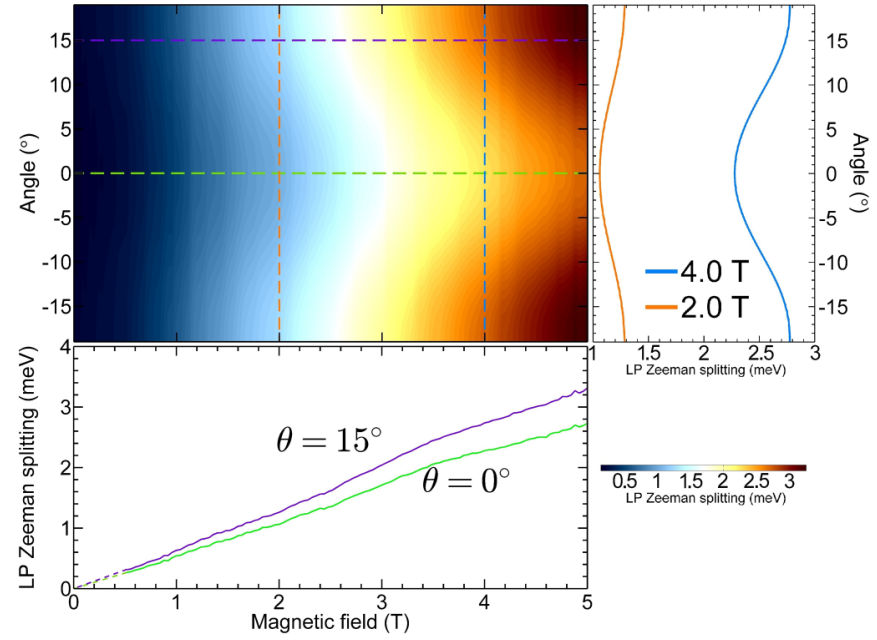

FIG. 5. Map of the lower polariton Zeeman splitting in a magnetic field for the results in positive detuning, $\delta_{0 \mathrm{~T}}=+1.3 \mathrm{meV}$. The righthand panel gives cross sections of the map for different values of magnetic field. The bottom panel shows cross sections for different angles of emission. The map color code illustrates the value of the Zeeman splitting of the lower polariton in $\mathrm{meV}$.

We attribute the loss of Rabi energy to the gradual transition to weak coupling of the individual exciton modes. As the magnetically induced broadening is increased, a fraction of localized exciton modes moves out of resonance and becomes weakly coupled to photons, which leads to the decrease of the overall coupling strength. The observed Rabi energy is therefore in our case an effective parameter taking into account coupling of many excitonic transitions with slightly different energy to a cavity mode. The images illustrating the excitonpolariton branches including the inhomogeneous broadening of the exciton line are presented in [43]. Such interpretation suggests that the value of the intrinsic Rabi coupling of individual exciton modes does not depend significantly on magnetic field but, due to the effect of inhomogeneous Mn distribution in QW, the effective Rabi energy changes.

The giant Zeeman effect of semimagnetic excitonpolaritons in a magnetic field is illustrated in Fig. 5. Based on the fitting of the solution of the Hamiltonian (1) to the experimental data for positive detuning illustrated in Fig. 2, we plot the energy difference between the two magnetically split lower polariton branches for different emission angles. The right-hand and bottom panels show cross sections of the polariton Zeeman splitting for two different emission angles and for two different values of magnetic field corresponding to the dashed lines marked on the map.

We observe that the lower polariton Zeeman splitting increases with emission angle, which indicates that the Zeeman splitting is higher for higher wave vectors. This effect results from the small effective mass of a microcavity photon compared to that of a QW exciton. Therefore, independently of the detuning at zero wave vector, at higher wave vectors the upper polariton state is more photonlike, whereas the lower polariton state is more excitonlike. Consequently, the lower polariton state is significantly more affected by a magnetic field $[14,16]$ at high wave vectors and the lower polariton energy splitting due to $s, p$ - $d$ exchange interaction is higher for high emission angles.

In the most general case, considering the coupling of the cavity photonic mode, $E_{\mathrm{ph}}$, to the heavy hole exciton in the QW, $E_{\text {exc }}$, and neglecting the coupling to the light hole exciton, the lower polariton energy in $\sigma^{+}$and $\sigma^{-}$polarizations can be expressed by the Hopfield coefficients in the following way:

$$
L P_{\sigma \pm}=\chi_{\sigma \pm}^{2} E_{\mathrm{exc}}^{\sigma \pm}+C_{\sigma \pm}^{2} E_{\mathrm{ph}}-\hbar \Omega \chi_{\sigma \pm} C_{\sigma \pm},
$$

where the $\chi^{2}$ and $C^{2}$ are the excitonic and photonic Hopfield coefficients, respectively [46]. These parameters give a direct measure of the excitonic and photonic content in the polariton state. The polariton Zeeman splitting, being the energy difference between $L P_{\sigma+}$ and $L P_{\sigma-}$, is therefore given by

$$
\begin{aligned}
\Delta E_{L P}= & \chi_{\sigma-}^{2} E_{\mathrm{exc}}^{\sigma-}-\chi_{\sigma+}^{2} E_{\mathrm{exc}}^{\sigma+}+\left(1-\chi_{\sigma-}^{2}\right) E_{\mathrm{ph}} \\
& -\left(1-\chi_{\sigma+}^{2}\right) E_{\mathrm{ph}}-\hbar \Omega\left(\chi_{\sigma-} C_{\sigma-}-\chi_{\sigma+} C_{\sigma+}\right)
\end{aligned}
$$

or, alternatively,

$$
\begin{aligned}
\Delta E_{L P}= & \chi_{\sigma-}^{2}\left(E_{\mathrm{exc}}^{\sigma-}-E_{\mathrm{ph}}\right)-\chi_{\sigma+}^{2}\left(E_{\mathrm{exc}}^{\sigma+}-E_{\mathrm{ph}}\right) \\
& +\hbar \Omega\left(\chi_{\sigma+} C_{\sigma+}-\chi_{\sigma-} C_{\sigma-}\right) .
\end{aligned}
$$

The polariton Zeeman splitting is therefore not directly proportional to the excitonic content in the polariton state, as can be well approximated for manganese-free GaAsbased structures [16]. The splitting is dependent on all three parameters (all influenced by the magnetic field): Hopfield coefficients, the photon-exciton detuning, and the Rabi coupling. In the case of polaritons composed of semimagnetic excitons, the excitonic Hopfield coefficient is significantly influenced by the magnetic field and the behavior is opposite for $\sigma^{+}$and $\sigma^{-}$polarizations, which is illustrated in Fig. 6(a) for zero and high emission angles, and for $2 \mathrm{~T}$ and $4 \mathrm{~T}$ in Fig. 6(b).

The linewidth in CdMnTe-based structures is broader than in nonmagnetic samples due the inhomogeneous $\mathrm{Mn}$ distribution. At zero magnetic field it is of $2.35 \mathrm{meV}$, but it decreases for $\sigma^{+}$to $2.2 \mathrm{meV}$ at $5 \mathrm{~T}$ and increases for $\sigma^{-}$ polarization to $2.5 \mathrm{meV}$. (The mechanism for narrowing and broadening of the polariton emission linewidth in magnetic field is described in [43].) The value of polariton Zeeman splitting was $\approx 3 \mathrm{meV}$ at $5 \mathrm{~T}$, at positive detuning at the temperatures of $10 \mathrm{~K}$. However, the polariton Zeeman splitting in our structure can be as high as $10 \mathrm{meV}$ at $5 \mathrm{~T}$ at a sufficiently low temperature of $1.4 \mathrm{~K}$, being much larger than the polariton linewidth. Nevertheless, even with the lower polariton linewidths comparable to the Zeeman splitting at $10 \mathrm{~K}$, separation of the emission lines with opposite circular polarizations is observable in the non-polarization-resolved experiment.

This is in contrast to the nonmagnetic GaInAs structures. Even though the polariton Zeeman splitting in GaInAs samples is of the order of $0.1 \mathrm{meV}$ at $5 \mathrm{~T}$, being comparable to or smaller than the polariton linewidth $(\approx 0.2 \mathrm{meV})[14,16]$, the significant diamagnetic shift in this material always results in the decrease of the exciton content for the lower polariton branch at high magnetic fields, which ends in the decrease of the observable polariton Zeeman splitting in the magnetic field. Therefore, even at a magnetic field of $14 \mathrm{~T}$ and at positive 

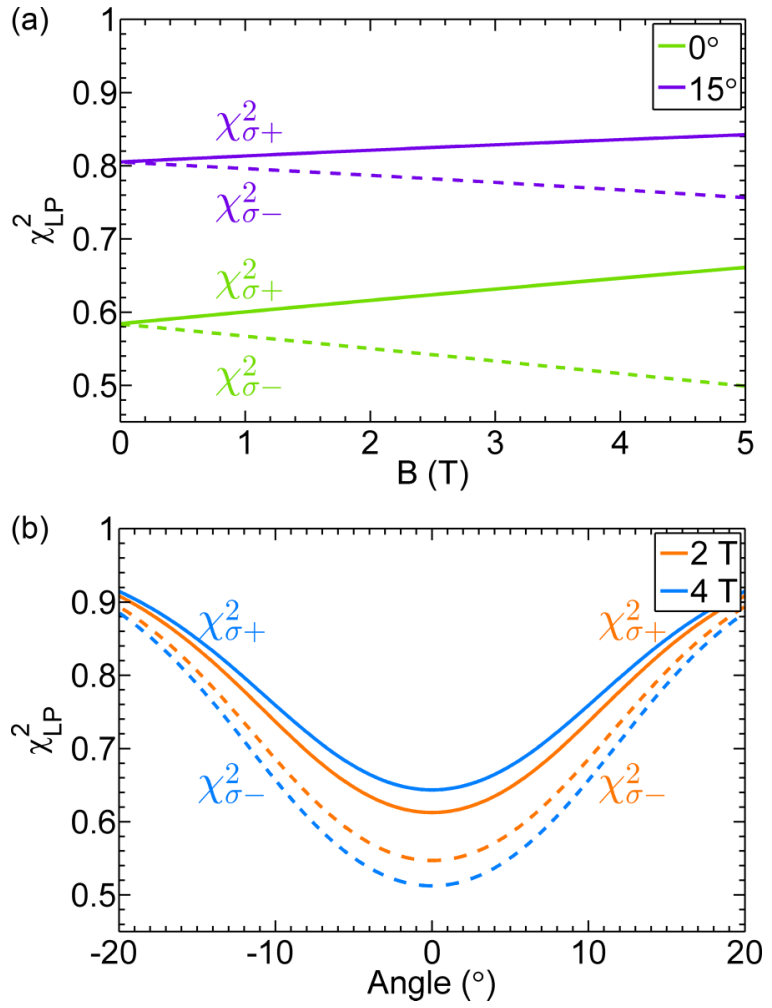

FIG. 6. (a) Evolution of the excitonic Hopfield coefficient, $\chi^{2}$, in a magnetic field for $\sigma^{+}$and $\sigma^{-}$polarized polaritons and for two different emission angles calculated for the detuning of $\delta_{0 \mathrm{~T}}=$ $+1.3 \mathrm{meV}$ at $0 \mathrm{~T}$. (b) Angular dependence of $\chi^{2}$ for $\sigma^{+}$and $\sigma^{-}$ polarized polaritons at magnetic fields of $2 \mathrm{~T}$ and $4 \mathrm{~T}$.

detuning, the polariton Zeeman splitting is not resolved from the polariton linewidth.

Since for $\mathrm{CdMnTe}$ at low fields diamagnetic shift is negligible compared to the giant Zeeman effect, the energy shift in the magnetic field for $\sigma^{+}$excitons leads directly to an increase of excitonic content $\left(\chi_{\sigma+}^{2}\right)$ in the polariton mode, as presented in Fig. 6(a).

The value of the observed polariton Zeeman splitting together with the absence of diamagnetic shift is remarkable and distinguishes our structure based on semimagnetic CdMnTe semiconductors from any other nonmagnetic microcavity sample. Moreover, the value of the Zeeman splitting can be even further increased with higher Mn content. The size of this effect is a direct consequence of the exchange interaction between delocalized $s$ - and $p$ - type band electrons and localized $d$-type manganese ions. Even if Zeeman splitting follows a similar law for nonmagnetic and magnetic samples its origin is different: for a nonmagnetic sample the splitting is caused by the magnetic field which acts on both (i.e., orbital and spin) parts of the wave function of the electron and hole, while in magnetic samples the splitting is due to exchange interaction, i.e., the "exchange magnetic field," which acts only on the spin part of the wave function. This means that the magnetic sample is not simply a nonmagnetic sample at very high magnetic field.

\section{SUMMARY}

We studied magnetic field effects on exciton-polaritons created in microcavities with semimagnetic quantum wells. The manganese ions incorporated into quantum wells enhance magnetic field effects by $s, p-d$ exchange interaction. We demonstrated that the exciton-polaritons formed from semimagnetic excitons exhibit giant Zeeman splitting in a magnetic field. We have shown that in these structures the magnetic field changes only the excitonic part of polaritons, and the splitting strongly depends on the photon-exciton detuning and polariton in-plane wave vector. Our results pave the way to the observation of many spin-related phenomena of excitonpolaritons in a magnetic field. The giant Zeeman splitting can be crucial in the observation of the spin Meissner effect [14] and spin-dependent polariton-polariton interactions [47]. Our results and the fabricated structure are the first step to explore complex phenomena in nonequilibrium spinor condensates of exciton-polaritons based on semimagnetic structures.

\section{ACKNOWLEDGMENTS}

This work was supported by the National Science Centre, Poland, under Projects No. 2014/13/N/ST3/03763, No. 2015/16/T/ST3/00506, No. 2015/18/E/ST3/00558, No. 2015/18/E/ST3/00559, No. 2013/09/B/ST3/02603, and No. 2015/17/B/ST3/02273, and by the Polish Ministry of Science and Higher Education as Iuventus Plus Research Grants No. IP2014 040473 and No. IP2014 034573 in years 2015-2017. This study was carried out with the use of CePT, CeZaMat, and NLTK infrastructures financed by the European Union, European Regional Development Fund. Scientific work was co-financed from the Polish Ministry of Science and Higher Education budget for education as a research project "Diamentowy Grant" No. 0010/DIA/2016/45 in years 2016-2020 and No. 0109/DIA/2015/44 in years 2015-2019.
[1] J. Kasprzak, M. Richard, S. Kundermann, A. Baas, P. Jeambrun, J. M. J. Keeling, F. M. Marchetti, M. H. Szymańska, R. André, J. L. Staehli, V. Savona, P. B. Littlewood, B. Deveaud, and Le Si Dang, Bose-Einstein condensation of exciton polaritons, Nature (London) 443, 409 (2006).

[2] R. Balili, V. Hartwell, D. Snoke, L. Pfeiffer, and K. West, Bose-Einstein condensation of Microcavity Polaritons in a trap, Science 316, 1007 (2007).

[3] A. Amo, J. Lefrère, S. Pigeon, C. Adrados, C. Ciuti, I. Carusotto, R. Houdré, E. Giacobino, and A. Bramati, Superfluidity of polaritons in semiconductor microcavities, Nat. Phys. 5, 805 (2009).

[4] G. Nardin, G. Grosso, Y. Léger, B. Pietka, F. Morier-Genoud, and B. Deveaud-Plédran, Hydrodynamic nucleation of quantized vortex pairs in a polariton quantum fluid, Nat. Phys. 7, 635 (2011).

[5] J. Levrat, R. Butté, E. Feltin, J.-F. Carlin, N. Grandjean, D. Solnyshkov, and G. Malpuech, Condensation phase diagram of cavity polaritons in GaN-based microcavities: Experiment and theory, Phys. Rev. B 81, 125305 (2010). 
[6] S. Klembt, E. Durupt, S. Datta, T. Klein, A. Baas, Y. Léger, C. Kruse, D. Hommel, A. Minguzzi, and M. Richard, ExcitonPolariton Gas as a Nonequilibrium Coolant, Phys. Rev. Lett. 114, 186403 (2015)

[7] T. C. H. Liew, I. A. Shelykh, and G. Malpuech, Polaritonic devices, Physica E 43, 1543 (2011).

[8] D. Ballarini, M. De Giorgi, E. Cancellieri, R. Houdré, E. Giacobino, R. Cingolani, A. Bramati, G. Gigli, and D. Sanvitto, All-optical polariton transistor, Nat. Commun. 4, 1778 (2013).

[9] A. V. Kavokin, M. R. Vladimirova, M. A. Kaliteevski, O. Lyngnes, J. D. Berger, H. M. Gibbs, and G. Khitrova, Resonant Faraday rotation in a semiconductor microcavity, Phys. Rev. B 56, 1087 (1997).

[10] G. Salis and M. Moser, Faraday-rotation spectrum of electron spins in microcavity-embedded GaAs quantum wells, Phys. Rev. B 72, 115325 (2005).

[11] D. D. Solnyshkov, M. M. Glazov, I. A. Shelykh, A. V. Kavokin, E. L. Ivchenko, and G. Malpuech, Magnetic field effect on polarization and dispersion of exciton-polaritons in planar microcavities, Phys. Rev. B 78, 165323 (2008).

[12] D. D. Solnyshkov, I. A. Shelykh, and G. Malpuech, Phase diagram of a spinor exciton-polariton condensate in a disordered microcavity in the presence of a magnetic field, Phys. Rev. B 80, 165329 (2009).

[13] A. V. Larionov, V. D. Kulakovskii, S. Höfling, C. Schneider, L. Worschech, and A. Forchel, Polarized Nonequilibrium BoseEinstein Condensates of Spinor Exciton Polaritons in a Magnetic Field, Phys. Rev. Lett. 105, 256401 (2010).

[14] P. Walker, T. C. H. Liew, D. Sarkar, M. Durska, A. P. D. Love, M. S. Skolnick, J. S. Roberts, I. A. Shelykh, A. V. Kavokin, and D. N. Krizhanovskii, Suppression of Zeeman Splitting of the Energy Levels of Exciton-Polariton Condensates in Semiconductor Microcavities in an External Magnetic Field, Phys. Rev. Lett. 106, 257401 (2011).

[15] J. Fischer, S. Brodbeck, A. V. Chernenko, I. Lederer, A. Rahimi-Iman, M. Amthor, V. D. Kulakovskii, L. Worschech, M. Kamp, M. Durnev, C. Schneider, A. V. Kavokin, and S. Höfling, Anomalies of a Nonequilibrium Spinor Polariton Condensate in a Magnetic Field, Phys. Rev. Lett. 112, 093902 (2014).

[16] B. Piętka, D. Zygmunt, M. Król, M. R. Molas, A. A. L. Nicolet, F. Morier-Genoud, J. Szczytko, J. Łusakowski, P. Zięba, I. Tralle, P. Stępnicki, M. Matuszewski, M. Potemski, and B. Deveaud, Magnetic field tuning of exciton-polaritons in a semiconductor microcavity, Phys. Rev. B 91, 075309 (2015).

[17] C. Sturm, D. Solnyshkov, O. Krebs, A. Lemaître, I. Sagnes, E. Galopin, A. Amo, G. Malpuech, and J. Bloch, Nonequilibrium polariton condensate in a magnetic field, Phys. Rev. B 91, 155130 (2015).

[18] V. P. Kochereshko, M. V. Durnev, L. Besombes, H. Mariette, V. F. Sapega, A. Askitopoulos, I. G. Savenko, T. C. Liew, I. A. Shelykh, A. V. Platonov et al., Lasing in Bose-Fermi mixtures, Sci. Rep. 6, 20091 (2016).

[19] J. A. Gaj, J. Ginter, and R. Gałąka, Exchange interaction of manganese $3 d^{5}$ states with band electrons in $\mathrm{Cd}_{1-x} \mathrm{Mn}_{x} \mathrm{Te}$, Phys. Status Solidi B 89, 655 (1978).

[20] J. A. Gaj, R. R. Gałazka, and M. Nawrocki, Giant exciton faraday rotation in $\mathrm{Cd}_{1-x} \mathrm{Mn}_{x}$ Te mixed crystals, Solid State Commun. 25, 193 (1978).
[21] J. Gaj, R. Planel, and G. Fishman, Relation of magnetooptical properties of free excitons to spin alignment of $\mathrm{Mn}^{++}$ions in $\mathrm{Cd}_{1-x} \mathrm{Mn}_{x}$ Te, Solid State Commun. 29, 435 (1979).

[22] J. Szczytko, W. Mac, A. Twardowski, F. Matsukura, and H. Ohno, Antiferromagnetic $p-d$ exchange in ferromagnetic $\mathrm{Ga}_{1-x} \mathrm{Mn}_{x}$ As epilayers, Phys. Rev. B 59, 12935 (1999).

[23] M. Poggio, R. C. Myers, N. P. Stern, A. C. Gossard, and D. D. Awschalom, Structural, electrical, and magneto-optical characterization of paramagnetic GaMnAs quantum wells, Phys. Rev. B 72, 235313 (2005).

[24] H. Ulmer-Tuffigo, J. Bleuse, F. Kany, R. André, and L. S. Dang, Magnetic tuning of exciton-photon resonance in II-VI microcavities, Superlattices Microstruct. 22, 383 (1997).

[25] M. Haddad, R. André, R. Frey, and C. Flytzanis, Enhanced Faraday rotation in an asymmetric semiconductor microcavity, Solid State Commun. 111, 61 (1999).

[26] D. Pereda Cubian, M. Haddad, R. André, R. Frey, G. Roosen, J. L. Arce Diego, and C. Flytzanis, Photoinduced magnetooptic Kerr effects in asymmetric semiconductor microcavities, Phys. Rev. B 67, 045308 (2003).

[27] M. Koba and J. Suffczyński, Magneto-optical effects enhancement in DMS layers utilizing 1-D photonic crystal, J. Electromagn. Wave 27, 700 (2013).

[28] M. Koba and J. Suffczyński, Angle dependence of photonic enhancement of magneto-optical Kerr effect in DMS layers, Europhys. Lett. 108, 27004 (2014).

[29] J. Sadowski, H. Mariette, A. Wasiela, R. André, Y. Merle d'Aubigné, and T. Dietl, Magnetic tuning in excitonic Bragg structures of (Cd,Mn)Te/(Cd,Zn,Mg)Te, Phys. Rev. B 56, R1664(R) (1997).

[30] A. Brunetti, M. Vladimirova, D. Scalbert, and R. André, Spin quantum beats in CdMnTe microcavity, Phys. Status Solidi C 2 , 3876 (2005).

[31] A. Brunetti, M. Vladimirova, D. Scalbert, M. Nawrocki, A. V. Kavokin, I. A. Shelykh, and J. Bloch, Observation of spin beats at the Rabi frequency in microcavities, Phys. Rev. B 74, 241101 (2006).

[32] A. Brunetti, M. Vladimirova, D. Scalbert, R. André, D. Solnyshkov, G. Malpuech, I. A. Shelykh, and A. V. Kavokin, Coherent spin dynamics of exciton-polaritons in diluted magnetic microcavities, Phys. Rev. B 73, 205337 (2006).

[33] W. Pacuski, T. Jakubczyk, C. Kruse, J. Kobak, T. Kazimierczuk, M. Goryca, A. Golnik, P. Kossacki, M. Wiater, P. Wojnar, G. Karczewski, T. Wojtowicz, and D. Hommel, Micropillar cavity containing a CdTe quantum dot with a single manganese ion, Cryst. Growth Des. 14, 988 (2014).

[34] J.-G. Rousset, J. Kobak, E. Janik, T. Jakubczyk, R. Rudniewski, P. Piotrowski, M. Ściesiek, J. Borysiuk, T. Slupinski, A. Golnik, P. Kossacki, M. Nawrocki, and W. Pacuski, MBE grown microcavities based on selenium and tellurium compounds, J. Cryst. Growth 401, 499 (2014).

[35] D. D. Solnyshkov, H. Flayac, and G. Malpuech, Stable magnetic monopoles in spinor polariton condensates, Phys. Rev. B 85, 073105 (2012).

[36] H. Ohadi, A. Dreismann, Y. G. Rubo, F. Pinsker, Y. del Valle-Inclan Redondo, S. I. Tsintzos, Z. Hatzopoulos, P. G. Savvidis, and J. J. Baumberg, Spontaneous Spin Bifurcations and Ferromagnetic Phase Transitions in a Spinor ExcitonPolariton Condensate, Phys. Rev. X 5, 031002 (2015). 
[37] J.-G. Rousset, J. Kobak, T. Slupinski, T. Jakubczyk, P. Stawicki, E. Janik, M. Tokarczyk, G. Kowalski, M. Nawrocki, and W. Pacuski, MBE growth and characterization of a II-VI distributed Bragg reflector and microcavity lattice-matched to MgTe, J. Cryst. Growth 378, 266 (2013).

[38] J.-G. Rousset, B. Piętka, M. Król, R. Mirek, K. Lekenta, J. Szczytko, J. Borysiuk, J. Suffczyński, T. Kazimierczuk, M. Goryca, T. Smoleński, P. Kossacki, M. Nawrocki, and W. Pacuski, Strong coupling and polariton lasing in Te based microcavities embedding $(\mathrm{Cd}, \mathrm{Zn}) \mathrm{Te}$ quantum wells, Appl. Phys. Lett. 107, 201109 (2015).

[39] A. V. Kavokin, J. J. Baumberg, G. Malpuech, and F. P. Laussy, Microcavities, Series on Semiconductor Science and Technology (Oxford University Press, New York, 2009).

[40] G. Bastard, E. E. Mendez, L. L. Chang, and L. Esaki, Exciton binding energy in quantum wells, Phys. Rev. B 26, 1974 (1982).

[41] E. L. Ivchenko, A. V. Kavokin, V. P. Kochereshko, G. R. Posina, I. N. Uraltsev, D. R. Yakovlev, R. N. Bicknell-Tassius, A. Waag, and G. Landwehr, Exciton oscillator strength in magnetic-fieldinduced spin superlattices CdTe/(Cd,Mn)Te, Phys. Rev. B 46, 7713 (1992).
[42] Q. X. Zhao, M. Oestreich, and N. Magnea, Electron and hole g-factors in CdTe/CdMgTe quantum wells, Appl. Phys. Lett. 69, 3704 (1996).

[43] See Supplemental Material at http://link.aps.org/supplemental/ 10.1103/PhysRevB.95.085429 for details of the fitting procedure to the experimental data and model of inhomogeneous exciton-polariton broadening in magnetic field in CdMnTebased microcavities.

[44] A. V. Komarov, V. I. Sugakov, G. V. Vertsimakha, W. Zaleszczyk, W. Karczewski, and T. Wojtowicz, Magnetic field dependence of exciton linewidth in quantum wells made of semimagnetic semiconductors: Comparison of theory and experiment, J. Phys.: Condens. Matter 18, 7401 (2006).

[45] V. I. Sugakov and G. V. Vertsimakha, Magnetic field dependence of the exciton bandwidth in diluted magnetic semiconductors with quantum wells, J. Phys.: Condens. Matter 13, 5635 (2001).

[46] J. J. Hopfield, Theory of the contribution of excitons to the complex dielectric constant of crystals, Phys. Rev. 112, 1555 (1958).

[47] T. Paraïso, M. Wouters, Y. Léger, F. Morier-Genoud, and B. Deveaud-Plédran, Multistability of a coherent spin ensemble in a semiconductor microcavity, Nat. Mater. 9, 655 (2010). 